\title{
Compositional variation of indentation-induced deformation and cracking in glass
}

\author{
Satoshi YOSHIDA, ${ }^{1, \dagger}$ \\ ${ }^{1}$ Glass and Ceramics Team, Inorganic Materials Division, Materials Integration Laboratories, AGC Inc., \\ 1150 Hazawa-cho, Kanagawa-ku, Yokohama 221-8755, Japan
}

The indentation test using a sharp diamond indenter, such as a Vickers indenter, has been long employed for comparing mechanical properties among various glass compositions, because of its simple procedure and easy operation. However, mechanisms of permanent deformation and crack nucleation in glass are far from simple. This has made it difficult to understand what controls the threshold load for cracking in a certain glass during indentation. In this review, the author's works on indentation-induced deformation and cracking in glass are introduced, and relevant issues on the indentation technique are discussed. Especially, it is pointed out that permanent densification of glass under the indenter is a key phenomenon which controls the following cracking event. Further, it is also shown that the micro-photoelastic observation system, by which the indentation-induced stress field can be visualized, and the indentation microscope, which enables one to measure the contact area between glass and the indenter, are powerful tools to stimulate new and fresh ideas for improving mechanical properties of glass. (O2020 The Ceramic Society of Japan. All rights reserved.

Key-words : Indentation, Hardness, Crack, Fracture, Densification, Glass

[Received April 27, 2020; Accepted May 18, 2020]

\section{Introduction}

It was over 70 years ago that Taylor first reported permanent imprints on glass surface after Vickers indentation. ${ }^{1)}$ He also noted that the size of the imprint varied with glass composition. This was the first report on compositional variation in hardness of glass. Although Taylor did not state about why glass behaved plastically under the indenter, other researchers tried to find out the origin of the plasticity of glass. After 19 years from the Taylor's note, Ernsberger proposed from his interferometric observation that hardness of glass did not have the same meaning as hardness of metals, and that hardness number of glass was a measure of hydrostatic pressure (sometimes assisted by shear stress) for initiating permanent densification. ${ }^{2)}$ It is not surprising that this hypothesis was able to be led by the discovery of permanent densification of glass under high hydrostatic pressure. ${ }^{3)}$ At that time, however, it remained unclear how shear stress under the sharp indenter affected the indentation-induced deformation, or hardness, of glass. After two years from Ernsberger's hypothesis, Peter reported that not only permanent densification but shear flow occurred in oxide glass under a sharper diamond indenter, and that deformation mechanism, densification or shear flow, in glass under a given indenter varied with

\footnotetext{
Corresponding author: S. Yoshida;

E-mail: satoshi.s.yoshida@agc.com
}

glass composition. ${ }^{4)}$ As evidences of shear flow in glass, he showed the pile-up around the indentation imprint and slip-lines which are curved lines developed under a contact region between glass and the indenter. In his paper, Peter concluded that the permanent densification under the indenter was a more general property of glasses, whereas shear flow in glass at room temperature seemed to require a minimum percentage of network modifiers. According to this Peter's finding, Yamane and Mackenzie proposed an equation for direct calculation of Vickers hardness of glass from its chemical composition. ${ }^{5)}$ Their method is based on a consideration of three kinds of deformation modes during indentation, which are elastic deformation, shear flow deformation, and permanent densification. They assumed that Vickers hardness of glass represented the resistance to these deformations, all of which can be related to elastic moduli of the glass. The model proposed by Yamane and Mackenzie is very suggestive with regard to the origin of plastic deformation in glass. The reasonable agreement between their model and experimental data suggests that plastic deformation in glass may be in an elastically-stressed and frozen-in state. In order to learn more about the plasticity of glass not only under the indenter but under various loading conditions, one can refer to the chapter of the recently published book and to the references in it. ${ }^{6}$ )

Following the pioneering works stated above, Arora et al. reported that different crack patterns around the 
indentation imprint originated from different deformation mechanisms. ${ }^{7}$ Anomalous glass like silica or Pyrex glass deforms predominantly by permanent densification, whereas normal glass like soda-lime glass deforms by both densification and shear flow. This is in line with Peter's finding. The different deformation mechanisms result in different residual stress fields to leave different crack patterns. It may be not surprising, but Arora's paper reminds us that indentation deformation is followed by cracking, or brittle behavior of glass. Not only experimental works but analytical models ${ }^{8)-10)}$ promoted better understanding of the indentation-induced cracking. Actually, Cook and Pharr ${ }^{11)}$ reported that Yoffe's model ${ }^{8)}$ successfully reproduced dynamic cracking sequences and crack geometries for some glasses (four types of glasses) during the indentation loading-unloading cycle. However, detail compositional variation of the driving force for cracking was unclear, although it was known that crack initiation load of glass during Vickers indentation varied distinctly with glass composition. ${ }^{12)}$ Nevertheless, the indentation test is still one of the simplest solutions in order to compare damageability among glass compositions.

In this review, the author introduces some techniques to evaluate compositional variation of the indentationinduced deformation and cracking in glass, especially in oxide glass. These techniques include a novel in-situ evaluation method to determine the indentation-induced stress field in glass and an in-situ observation set-up to determine the contact area and the cracking sequence of glass under the indenter. In addition, a future perspective to obtain deeper insight into brittleness of glass is also surveyed.

\section{Classification of densification and shear flow in glass under an indenter}

As stated above, it has been reported that the deformation mechanism of glass under an indenter depends on glass composition. It also affects subsequent cracking behavior. Therefore, in order to understand the origin of the compositional variation of cracking in glass, we should focus closely on the deformation mechanism, which is one of the fracture precursor phenomena.

It is known that an indentation imprint on glass can be partially recovered by thermal annealing at around its glass transition temperature. ${ }^{13)-15)}$ The amount of thermal shrinkage of the indentation imprint depends on glass composition, and the shrinkage process results from volume relaxation of densified glass. ${ }^{16)}$ However, the previous studies $^{13)-15)}$ focused only on one-dimensional (the diagonal length of the imprint) shrinkage. Using an atomic force microscope (AFM) or a scanning probe microscope, one can determine the volume difference in an indentation imprint before and after annealing. ${ }^{17)}$ The present author assumed that the volume difference corresponds to the densified volume during indentation. The indentation lost volume depends on glass hardness. The smaller indentation volume means the larger hardness. Therefore, in order to compare the sensitivity to densification of glasses with different compositions, the present author proposed a parameter, which is the ratio of the recovered volume by annealing to the initial indentation lost volume. ${ }^{17)}$ This parameter represents the densification-contribution of a given glass under the indenter. It is also noted that indenter geometries, such as the tip-angle or the number of ridges, change the densification-contribution of a given glass. ${ }^{18)}$

The annealing of the indented glass was performed in air. ${ }^{17)}$ The annealing temperature and time were determined to be $\mathrm{Tg} \times 0.9(\mathrm{~K})$ and $2 \mathrm{~h}$, respectively, where $\mathrm{Tg}$ is glass transition temperature. Because viscous flow kinetics for silicate glass at the annealing temperature is very slow in comparison to the experimental time, ${ }^{17)}$ one can ignore the viscous flow during the annealing. In addition, the annealing time is long enough to achieve the complete recovery of the densified region. These experimental conditions were derived from the annealing behavior of a hydrostatically densified silica glass. ${ }^{16)}$ Of course, however, relaxation kinetics of the densified glass (the indented glass) should depend on the glass composition, or on the temperature-viscosity relation of the glass. Therefore, $\mathrm{Tg}$ $\times 0.9(\mathrm{~K})$ and $2 \mathrm{~h}$ should be regarded as one proposed condition in order to compare the densification-contribution among various glass compositions.

Before and after annealing, geometries of the indentation imprint were measured by using AFM. The procedure is schematically represented in Fig. 1. The volume ratio of annealing recovery, $V_{R}$, is calculated by the following equation, ${ }^{17)}$

$$
V_{\mathrm{R}}=\frac{\left(V_{\mathrm{i}}^{-}-V_{\mathrm{a}}^{-}\right)+\left(V_{\mathrm{a}}^{+}-V_{\mathrm{i}}^{+}\right)}{V_{\mathrm{i}}^{-}},
$$

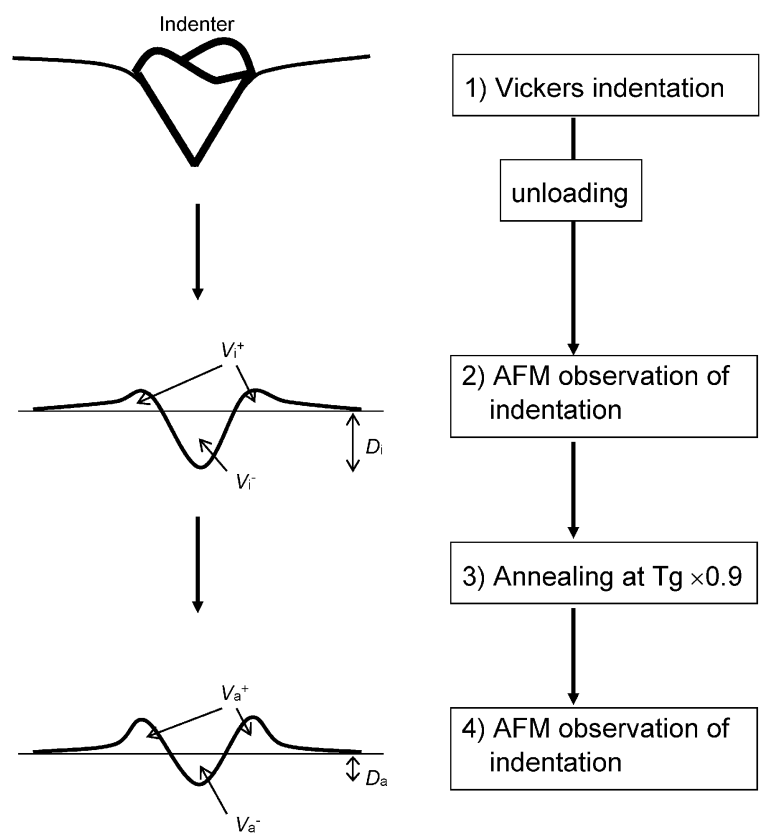

Fig. 1. Schematic procedure for measuring the indentation volume before and after annealing. $V_{\mathrm{i}}^{-}, V_{\mathrm{i}}^{+}, D_{\mathrm{i}}$ are the indentation lost volume, the pile-up volume, the indentation depth before annealing, respectively. $V_{\mathrm{a}}^{-}, V_{\mathrm{a}}^{+}, D_{\mathrm{a}}$ are the indentation volume, the pile-up volume, the indentation depth after annealing, respectively. This figure is reproduced from Ref. 17. 


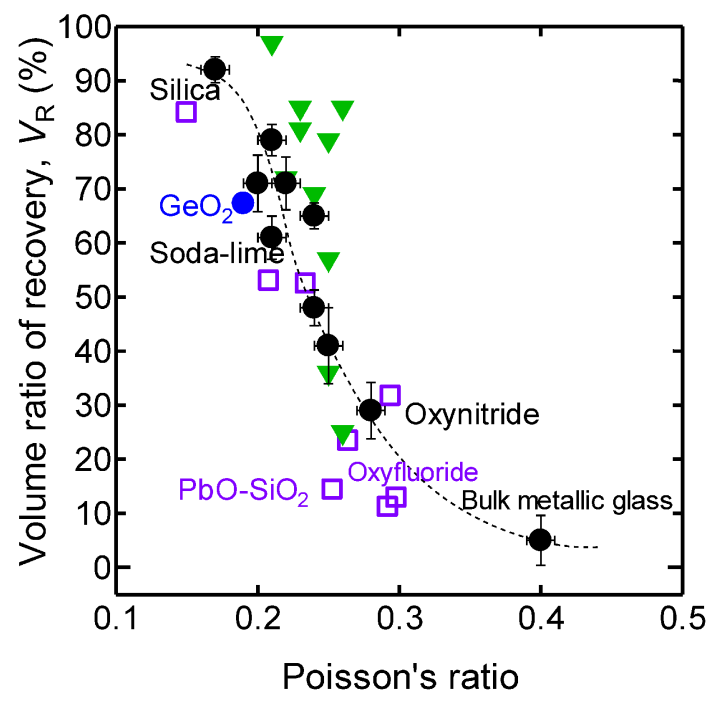

Fig. 2. A relation between Poisson's ratio and the volume ratio of annealing recovery, $V_{R}$. The dotted line is a guide for the eyes. Black circles, purple squares, and green triangles are obtained from Refs. 17, 20, and 21, respectively. The unpublished data point of $\mathrm{GeO}_{2}$ glass is also plotted as a blue circle. This figure is reproduced from Ref. 22.

where $V_{\mathrm{i}}^{-}$is the indentation lost volume before annealing, $V_{\mathrm{a}}^{-}$is the indentation volume after annealing, $V_{\mathrm{i}}^{+}$is the pile-up volume before annealing, and $V_{\mathrm{a}}^{+}$is the pile-up volume after annealing. It is assumed that both changes in the pile-up, $\left(V_{\mathrm{a}}^{+}-V_{\mathrm{i}}^{+}\right)$, and in the indentation volumes, $\left(V_{\mathrm{i}}^{-}-V_{\mathrm{a}}^{-}\right)$, are constituent of the total densified volume, and that the initial pile-up, $V_{i}^{+}$is formed during the unloading cycle, not during the loading cycle. In other words, $V_{i}^{+}$ is a part of the compressed volume at a maximum load. Since the densified volume is regarded as a part of the indentation lost volume, $V_{\mathrm{R}}$ should be the ratio of the recovered volume to the initial indentation volume, $V_{\mathrm{i}}^{-}$, not the ratio of the recovered volume to $\left(V_{i}^{-}-V_{i}^{+}\right)$. For some silicate glasses, the assumption that $V_{\mathrm{i}}^{+}$is formed during the unloading cycle was confirmed experimentally from in-situ observation by using an indentation microscope, ${ }^{19)}$ but it may not be the case for much softer glasses.

Figure 2 shows a relation between the volume ratio of densification for Vickers indentation and Poisson's ratio of glass. Although the data points are scattered, the volume ratio of densification, or the densification-contribution, has a tendency to decrease with increasing Poisson's ratio. This means that higher ability of a glass to exhibit volume change in the elastic regime (lower Poisson's ratio) would result in higher sensitivity to permanent densification under a Vickers indenter. However, it is noted that not only Poisson's ratio controls the densification of glass under the indenter. Some borate and borosilicate glasses, in which oxygen coordination number of boron would increase under high pressure or under a sharp indenter, show higher values of the densification-contribution than that estimated from their Poisson's ratios. ${ }^{23)-26)}$

As Yoffe's model showed, ${ }^{8)}$ the indentation-induced densification improves the resistance to median/radial

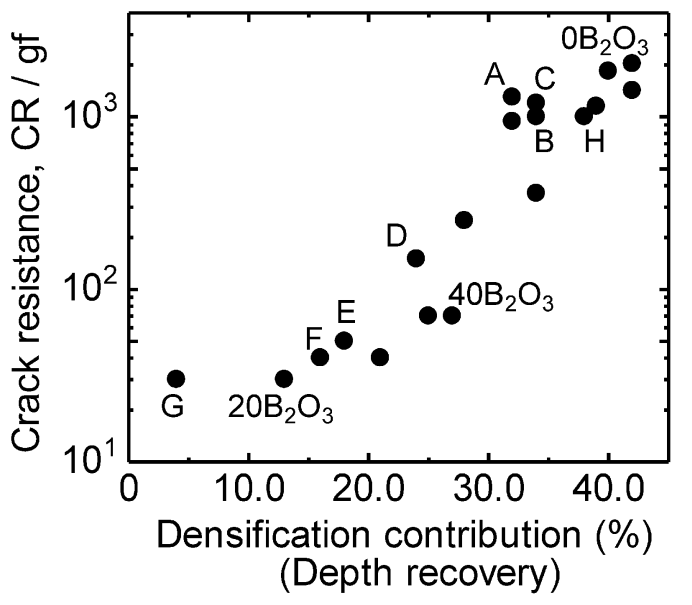

Fig. 3. A relation between the $\mathrm{CR}$ and recovery of indentation depth. The labels of $\mathrm{A}$ to $\mathrm{H}$ denote commercial glass compositions. The legends of $0 \mathrm{~B}_{2} \mathrm{O}_{3}, 20 \mathrm{~B}_{2} \mathrm{O}_{3}$, and $40 \mathrm{~B}_{2} \mathrm{O}_{3}$ denote glass compositions of $20 \mathrm{Na}_{2} \mathrm{O}-80 \mathrm{SiO}_{2}, 20 \mathrm{Na}_{2} \mathrm{O}-20 \mathrm{~B}_{2} \mathrm{O}_{3}-60 \mathrm{SiO}_{2}$, and $20 \mathrm{Na}_{2} \mathrm{O}-40 \mathrm{~B}_{2} \mathrm{O}_{3}-40 \mathrm{SiO}_{2}(\mathrm{~mol} \%)$, respectively. The other data points represent other borosilicate glass compositions. All the data are obtained from Refs. 27 and 28. This figure is reproduced from Ref. 22.

cracking during the indentation cycle. According to her model, densification reduces the driving force for median/ radial cracking to increase in crack initiation load. Figure 3 shows the experimental confirmation of her model. With increasing the depth ratio of indentation impression, which also corresponds to the densificationcontribution, the crack initiation load increases. In Fig. 3, the crack initiation load is defined as the load where the crack forming probability after Vickers indentation is $50 \% .^{27), 28)}$ Similar relations between the densificationcontribution and the crack initiation load have been reported. ${ }^{20), 25), 29)}$ However, various counter-examples of this relation were also reported (See the Ref. 24). This is probably due to coordination change of network forming cations during indentation and/or different intrinsic strength in glass. The relation shown in Fig. 3 implies that the densificationcontribution of glass under the indenter is one of various controlling factors for median/radial cracking in the glass.

The indentation test has been long used to mimic a contact damage in glass against collision of a foreign body. This is primary because the Vickers indentation test can create median/radial cracks which propagate perpendicular to a glass surface, and one of which would be the fracture origin. Figure 3 suggests that the high-crack-resistant glass with a high crack initiation load stems from its high sensitivity to permanent densification under the indenter. However, shear flow is another energy-dissipated process during the indentation. The mechanism of shear flow in oxide glass is still unclear, although some challenging approaches are now ongoing. For example, Martinet et al. pointed out that shear stress promoted an increase in the relative proportion of 3-membered $\mathrm{Si}-\mathrm{O}$ rings in silica glass, which are highly distorted structure in the glass. ${ }^{30}$ Furthermore, Rountree et al. and Sato et al. reported that a 


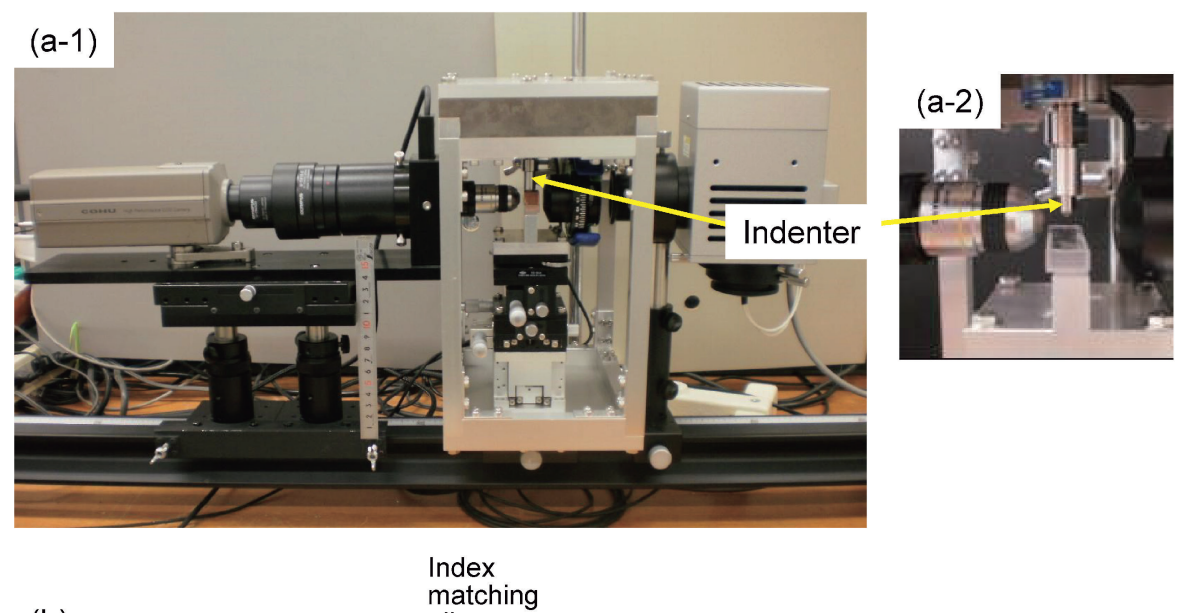

(b)

oil

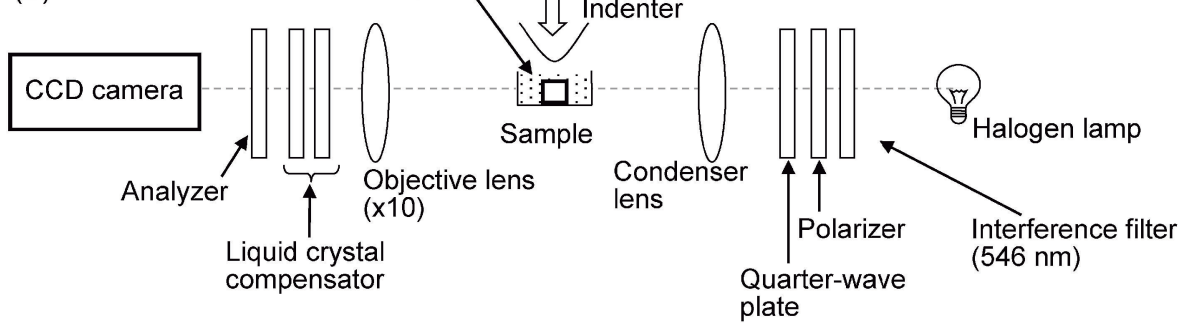

Fig. 4. Photos and schematic drawing of the experimental set-up for birefringence measurements during and after indentations. (a-1) and (a-2) are a photo of the set-up and a close-up picture around the indenter, respectively. (b) is a schematic drawing of the set-up. The figure (b) is reproduced from Ref. 41.

permanent anisotropy was created and frozen-in under shear flow on silica glass. ${ }^{31), 32)}$ It is also noteworthy that amorphous $\mathrm{Al}_{2} \mathrm{O}_{3}$ thin-film can permanently deform under mixed shear and compression loading at room temperature. ${ }^{33)}$ These reports imply that a variety of metastable structures in amorphous oxide under shear may also control a threshold condition for cracking. A much better understanding of shear flow in glass will help one to make a correct interpretation of complicated compositional variation of indentation cracking.

\section{Quantitative evaluation of the indentation- induced stress field during the indentation}

As shown in the previous section, driving force for median/radial cracking is effectively reduced by permanent densification of glass under the indenter. However, there have been very little experimental reports available for quantitative evaluation of the indentation-induced stress field in glass. This is because stress determination of the indented glass is still a challenging task. Furthermore, the cracking in glass occurs during indentation, not after indentation (, although sometimes we can see pop-in cracking after complete unload). Thus, we should focus on a dynamic change in stress distribution during indentation.

There are two successful examples for determining the stress field around the indentation imprint. One is the double-indentation technique, in which small cracked indentations are used as a stress prove on surface, ${ }^{34)-36)}$ and the other is the cathodoluminescence technique, in which piezo-spectroscopy assessments are applied to obtain a stress map on surface. ${ }^{37)}$ These techniques have advantages in some cases, but cannot be applicable to in-situ measurements of stress field during indentation and to measurements of interior stresses of glass under the indenter. One important solution is a birefringence technique, which uses artificial double refraction of a stressed glass. This technique has a long history. In 1922, for example, Dalladay and Twyman reported the tensile and compressive stressed regions below a scribe line on a glass plate by using the birefringence technique. ${ }^{38)}$ They discovered the localized tensile stress just below the scribe line, which would be the driving force for median cracking. Of course, however, the limited spatial resolution of their technique at that time did not permit evaluation of the stress field around an uncracked indentation imprint.

With an aid of the integrated photoelasticity ${ }^{39)}$ and a liquid-crystal compensator, ${ }^{40)}$ nowadays, stress components around a small imprint can be determined from retardation and azimuth of transmitted polarized light. If we use an axisymmetrical indenter like a spherical or a conical indenter, we can obtain a 3-dimensional stress map of the indented glass with a high spatial resolution of $\sim 1$ $\mu{ }^{41), 42)}$ Figure 4 shows photos and a schematic drawing of the birefringence measurement system. ${ }^{41)}$ A square glass fiber with a dimension of $0.5 \times 0.5 \times 20 \mathrm{~mm}^{3}$ was placed in a transparent boat in which the index matching oil was filled. During indentation, retardation and azimuth (slow axis orientation) were measured using the LCPolScope system ${ }^{40}$ including a charge coupled device (CCD) camera, a liquid-crystal compensator, an analyzer, a 
Distance from the loading axis, $r / \mu \mathrm{m}$

\section{Distance from the loading axis, $r / \mu \mathrm{m}$}
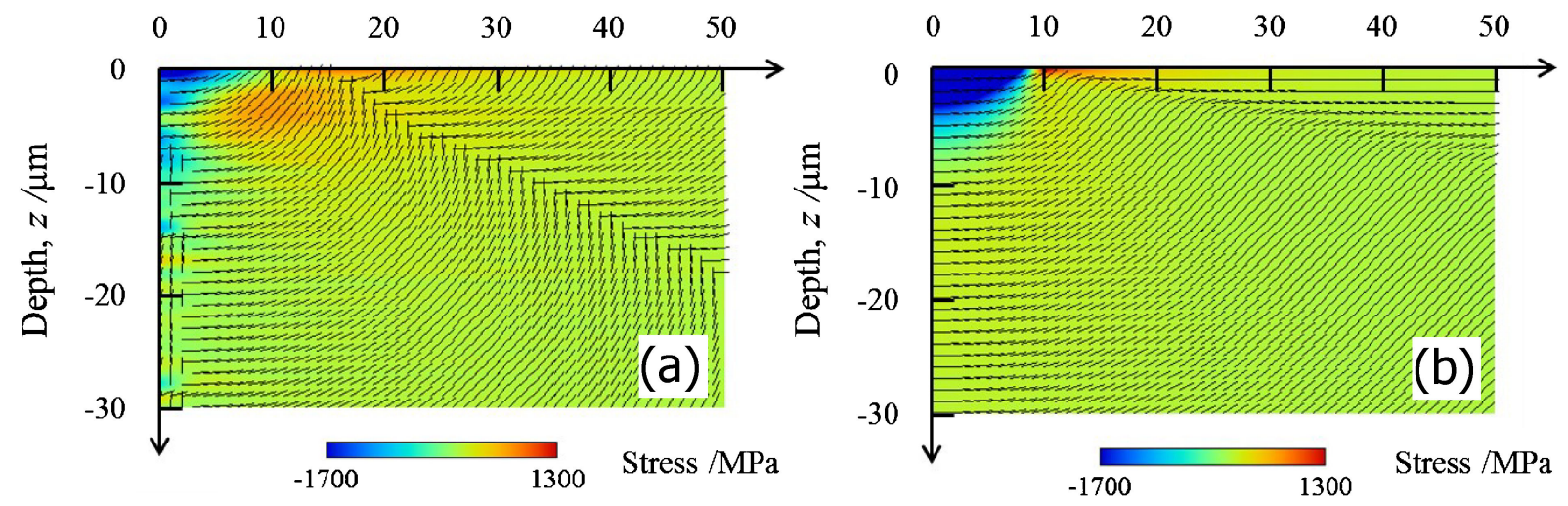

Fig. 5. Stress maps of maximum principal stress of silica glass during a ball indentation at $1.5 \mathrm{~N}$. The radius of the diamond ball indenter is $0.05 \mathrm{~mm}$. Birefringence stresses and Hertzian stresses are shown in (a) and (b), respectively. Fine black lines in the maps represent the direction of the principal stress.

quarter-wave plate, a polarizer, and an interference filter. The indentation load can be controlled using the z-stage and monitored with a load cell.

Figures 5(a) and 5(b) show an experimentally determined "elastic" stress field of silica glass (Young's modulus and Poisson's ratio are $73 \mathrm{GPa}$ and 0.17 , respectively.) under a diamond ball indenter (Its radius is $0.05 \mathrm{~mm}$.) and a corresponding analytical stress field using Herzian equations, ${ }^{41,42)}$ respectively. In Figs. 5(a) or 5(b), the coordinate origin (the upper left corner) corresponds to the contact point. The Hertzian principal stresses and their trajectories were calculated using the values of Young's modulus $(1141 \mathrm{GPa})$ and Poisson's ratio (0.07) of the diamond indenter. The birefringence stress map agrees, to some extent, with the analytical solution. In Fig. 5(b), the tensile stressed region (the red region) is found on a sample surface near the contact edge, which is about $\mathrm{r}=9 \mu \mathrm{m}$ from the Hertz equation. This surface-localized tensile stress is also observed in the experimental result [Fig. 5(a)], and it actually causes ring/cone cracking. ${ }^{41), 42)}$

Although the birefringence technique is straightforward and effective to evaluate the stress field around the contact point, it includes a serious issue of the algorithm used. At a present stage, stress components are assumed to satisfy linear elastic constitutive equations. This means that much care should be taken if one wants to determine residual stresses or stresses during elasto-plastic deformation. The issue has not been solved yet, but we can compare the stress fields among glass compositions if the stresses are predominantly elastic. The present author and his colleagues reported the estimated principal stress maps of silica and soda-lime glasses during both loading and unloading at a load of $0.5 \mathrm{~N}^{42)}$ Although the stress maps during loading looked like each other, the stress maps during unloading, which were affected by plastic deformation, were different between two glasses, because the mechanisms of plastic deformation are different. Silica glass prefers densification, whereas soda-lime glass does shear flow, as stated above. Therefore, it is highly expected that future studies will improve the algorithm to obtain much more quatitative stress fields of plastically deformed glasses.

\section{In-situ evaluation of mechanical responses of glass by using an indentation microscope}

The indentation-induced cracking is always accompanied with elastic or elasto-plastic deformation. In other words, the deformation mechanism affects the driving force for cracking. Therefore, in order to know when, where, how a crack nucleates during the indentation, direct observation of a contact region between glass and the indenter would be indispensable. However, there have been limited number of researches focusing on in-situ observation of elasto-plastic deformation of glass under the indenter. Lawn et al. first reported that the in-situ observation apparatus enabled one to observe the crack pop-in kinetics. ${ }^{43), 44)}$ Following these works, Cook and Pharr reported cracking sequences and crack geometries of some glasses and transparent ceramics under a Vickers indenter by using their observation system, ${ }^{11)}$ and concluded that the cracking sequence during an indentation cycle can be predicted from an indentation-induced stress field which depends on a modulus-to-hardness ratio (or elasticity index shown below) and (in the case of glass) on the contribution of permanent densification under the indenter. Such an in-situ observation apparatus is useful not only for evaluation of the cracking kinetics among various glasses and ceramics, but for evaluation of a real contact area during indentation. ${ }^{45), 46)}$

The contact area at a given load is important to evaluate in-situ hardness, or contact pressure, and indentation modulus of a material. This is because the contact pressure is directly linked to the indentation-induced stress field. For depth-sensing indentation, the contact area during indentation is estimated from an indentation depth using a calibration procedure, not from direct observation. The most popular procedure to estimate the contact area from the indentation depth is the one proposed by Oliver and 

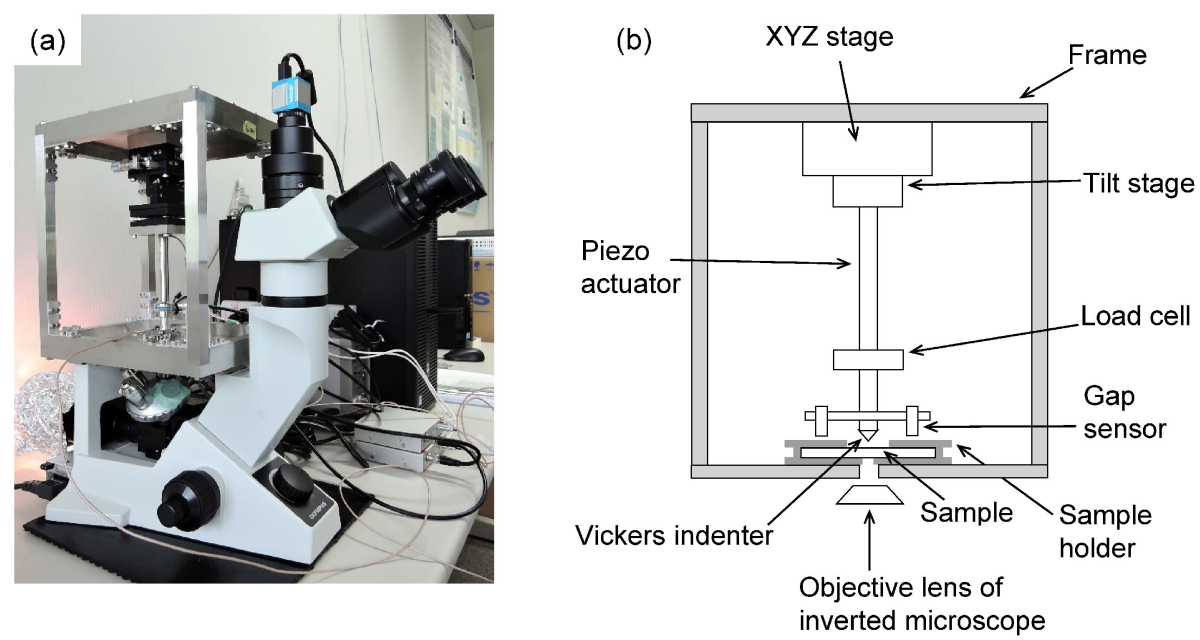

Fig. 6. Photo (a) and schematic drawing (b) of the indentation microscope. In the photo (a), the indentation system is placed on the stage of the inverted microscope. The figure (b) is reproduced from Ref. 48.

Pharr. ${ }^{47)}$ For glass mechanists, however, a serious issue is that no one knows any effects of densification deformation on the estimation procedure of the contact area. Therefore, determination of the true contact area is indispensable for estimating the contact stress of glass during indentation. Although the previous researchers took oxide glass as one of ceramic materials with high elasticity indices, $H / E$ (, where $H$ and $E$ are hardness and Young's modulus respectively), compositional variation of mechanical responses of oxide glass under the indenter must be focused on in order to understand the origin of wide variety of brittle responses of glass.

Figure 6 shows a photo and a schematic drawing of the self-made indentation microscope. ${ }^{48)}$ A commercial inverted microscope was used in order to observe the indentation sequence from below. A Vickers indenter was driven at a constant rate by using a piezo-actuator. The indentation load was monitored with a load cell. In order to obtain much clearer images with a higher resolution, it was indispensable to use an objective lens with an aberration correction ring. This objective lens is the one designed in order to observe specimens (for example, liquid crystals) through a glass substrate. The image can be directly recorded by a CCD camera in the bright field. In addition, the indentation depth, which is the position of the tip of Vickers indenter from a sample surface, can be measured by averaging the sensing values of two capacitive air gap sensors.

Figure 7 shows relations between indentation load and in-situ contact area for three kinds of silicate glasses. In order to reduce cracking, which disturbs determination of the contact area, the indentation test was performed under $\mathrm{N}_{2}$ flow. From the slope of the loading curve for each glass, "in-situ" Meyer hardness, which is the load divided by the in-situ projected contact area, can be calculated. In-situ Meyer hardness of silica, soda-lime, and lead silicate glasses are 7.4, 5.8, and 4.7 GPa, respectively. Linear relations on loading shown in Fig. 7 confirm that hardness of elasto-plastic material such as glass is a measure of

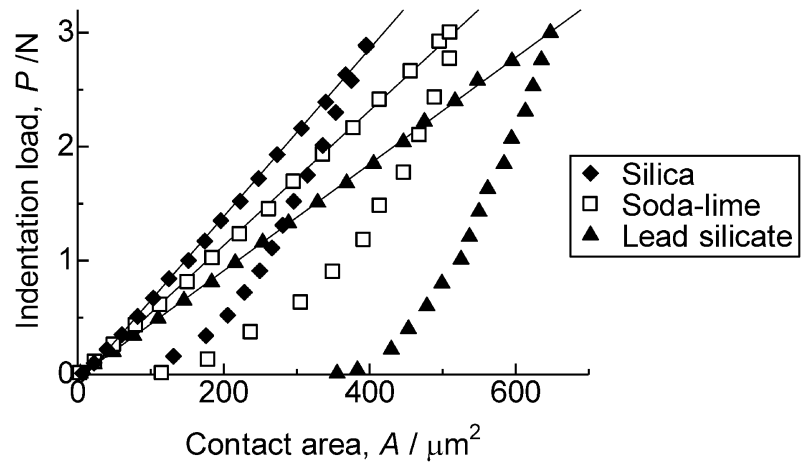

Fig. 7. Relations between the indentation load and the in-situ contact area for three kinds of silicate glasses under $\mathrm{N}_{2}$ flow (Closed diamond: Silica glass, Open square: Soda-lime glass, Closed triangle: Lead silicate glass). The solid lines are least square fits of the loading curves to linear functions. This figure is reproduced from Ref. 48.

resistance to elasto-plastic deformation, not resistance to plastic deformation, as suggested by Sakai. ${ }^{49)}$

Using the indentation microscope, it was found that a contact region between glass and a Vickers indenter was not a regular square but a concaved square, and that an amount of the bowed-in at a contact edge depended on glass composition and on indention load. ${ }^{48}$ As shown in Fig. 8(a-1), silica glass shows distinct bowed-in edges. The amount of the bowed-in parameter, which was proposed in the previous paper, ${ }^{50)}$ decreases with decreasing the elasticity index, $H / E$, at a given indentation load. ${ }^{48)}$ Lim and Chaudhri ${ }^{50)}$ attributed this bowing-in to pincushioning of an elastic body, where the depth of contact is greater at the ridges of the indenter than the depth of the points between ridges.

Because the sinking-in at the center between the corners causes a tensile stress in a radial direction at the indentation edge, the tensile stress may cause edge cracking along the edge of the indentation imprint. Depending on surface conditions and on testing environment, we can find the edge crack during both loading and unloading. ${ }^{48)}$ 

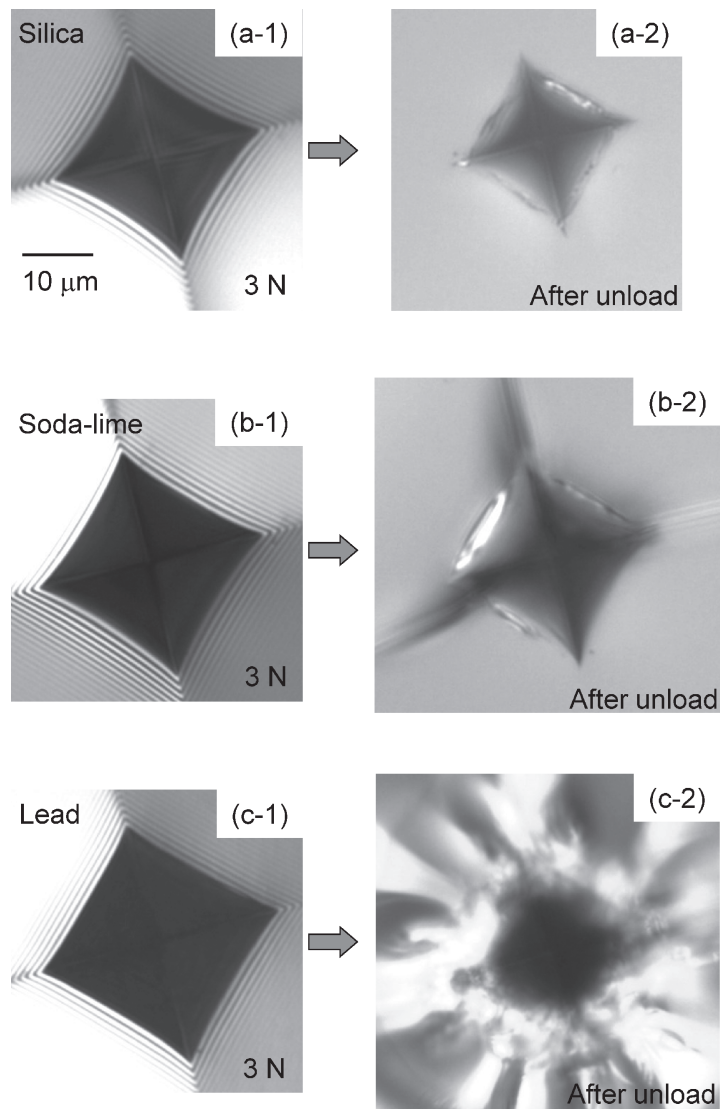

Fig. 8. Photos of the contact regions under load and the imprints after unload. The maximum indentation load is $3.0 \mathrm{~N}$. The indentations were performed under $\mathrm{N}_{2}$ flow. (a-1) Silica glass under a load of $3.0 \mathrm{~N}$, (a-2) Silica glass after complete unload, (b-1) Soda-lime glass under a load of 3.0 N, (b-2) Soda-lime glass after complete unload, (c-1) Lead silicate glass under a load of $3.0 \mathrm{~N}$, (c-2) Lead silicate glass after complete unload. All images have the same scale bar shown in (a-1).

The edge cracks are observed in Figs. 8(a-2) and 8(b-2). During loading, defects or faults are created in glass under the indenter, as pointed out by Lawn. ${ }^{51)}$ Depending on size and orientation, some defects or pre-existing defects may open into edge cracks during loading, ${ }^{48)}$ whereas other defects may turn into edge cracks during unloading, as shown in Figs. 8(a-2) and 8(b-2). It can be inferred that the driving force for the edge cracking during both loading and unloading is the tensile stress due to the sinking-in as stated above. Radial cracks can be found only in soda-lime and lead silicate glasses [Figs. 8(b-2) and 8(c-2)], because less densification-contribution of these glasses results in larger driving force for median/radial cracking, as stated above.

Another characteristic of the image obtained using the indentation microscope is existence of fringe patterns shown in Figs. 8(a-1), 8(b-1), and 8(c-1). The fringe patterns come from the interference of light waves reflected from both glass surface and the indenter. The interval between two black lines corresponds to the height difference of half a wavelength between the glass surface and the indenter. The quantitative determination of elastic deformation around the contact region was discussed elsewhere. ${ }^{52)}$

\section{Future perspective for development of high-crack-resistant glass}

Fracture in glass includes two events, crack initiation and crack propagation. The indentation-induced cracking should be closely related to the crack initiation. Although the crack propagation is controlled by fracture mechanics parameters, such as fracture toughness, there are still so many unknown issues on the crack initiation. Since the crack initiation is accompanied with dynamic structural changes in an atomistic level, we should focus on dynamic changes in glass structure just before the crack initiation. Some experimental researches are now in progress using various techniques, such as Raman spectroscopy including low-energy spectrum or Boson peak, ${ }^{53)}$ Brillouin spectroscopy,${ }^{54)}$ small angle X-ray scattering, ${ }^{55)}$ digital holographic tomography, ${ }^{56)}$ and X-ray tomography. ${ }^{57)}$

As for in-situ Raman spectroscopy of glass during the indentation, the present author and his colleagues fabricated a custom indenter for Raman measurements, and reported remarkable peak broadening of Raman spectrum of silica glass under a Vickers indenter. ${ }^{58)}$ The recent work ${ }^{59)}$ also showed the peak broadening of silica glass, although the authors of the paper alerted to the problem that the in-situ Raman spectra include spectral contribution of the indenter and that the ratio of the analyzed volume by Raman spectroscopy to the volume deformed by indentation must affect both the peak shape and the peak position. Nevertheless, the peak broadening is one of the characteristic features of glass under the indenter, because it cannot be observed in hydrostatic compressed silica glass under an elastic regime. ${ }^{60)}$ Such a transient structure in glass may control the crack initiation. Not only the structure but the stress field, or the driving force, should be the origin of the crack initiation. Furthermore, environmental conditions, such as relative humidity, also have an impact on the cracking event. Various types of new approaches including the modeling calculation will be required to obtain much deeper insight into the crack initiation in glass and to develop high-crack-resistant glass.

\section{Summary}

About 25 years ago, the author encountered some fundamental questions on the indentation cracking in glass. What controls the crack initiation load of glass under a Vickers indenter? Why lead-containing soft glass is so brittle, and why mother glass of glass-ceramics is so strong? Now, he has arrived at one key parameter, which is permanent densification of glass. Of course, it is not only permanent densification that controls the cracking behavior in glass. But, the permanent densification of some silicate glasses under the indenter reduces the driving force for median/radial cracking. There are several types of counter-examples. For example, glasses containing 5- or 6-coordinated aluminum ions have higher crack resistance (CR), but do not prefer permanent densification 
under the indenter. Fortunately, the counter-examples are now stimulating various discussion on the mechanism of the indentation-induced cracking in glass.

In-situ evaluation techniques of stress fields and cracking sequences are indispensable in order to compare brittleness among glass compositions. Two examples, which are the birefringence technique and the indentation microscope, are introduced in this review. These are based on classical techniques, but visualization of the controlling factors for cracking should promote various new ideas to develop less-brittleness glasses. Although the brittle behavior of glass has been long an unsolved problem, the author believes that new characterizing devices in microand nano-scopic scale, testing machines in nanometer scale, and brand-new modeling techniques will give us a new way to overcome the brittleness of glass.

Acknowledgements The author expresses his sincere appreciation to Professor Jun Matsuoka, Professor Akihiro Yamada, and all the former professors, and past and present staffs and students in Ceramic Materials Laboratory and in Center for Glass Science and Technology of The University of Shiga Prefecture, Japan, at which the author started his work on the indentation on glass and enjoyed it for more than 20 years. The author is also indebted to Mr. Masamichi Wada, Dr. Chuck Kurkjian, Dr. Munawar M. Chaudhri, Professor Tanguy Rouxel, Professor Jean-Christophe Sanglebœuf for encouraging him and stimulating discussions on this topic. Further, the author acknowledges continuous financial support by Nippon Electric Glass Co. Ltd., Japan and by JSPS KAKENHI (25420713 and 16K06730).

\section{References}

1) E. W. Taylor, Nature, 163, 323 (1949)

2) F. M. Ernsberger, J. Am. Ceram. Soc., 51, 545-547 (1968).

3) P. W. Bridgman and I. Šimon, J. Appl. Phys., 24, 405413 (1953).

4) K. W. Peter, J. Non-Cryst. Solids, 5, 103-115 (1970).

5) M. Yamane and J. D. Mackenzie, J. Non-Cryst. Solids, 15, 153-164 (1974).

6) J.-P. Guin and Y. Gueguen, "Springer Handbook of Glass", Ed. by J. D. Musgraves, J. Hu and L. Calvez, Springer Nature, Cham, Switzerland (2019) pp. 241251.

7) A. Arora, D. B. Marshall, B. R. Lawn and M. V. Swain, J. Non-Cryst. Solids, 31, 415-428 (1979).

8) E. H. Yoffe, Philos. Mag. A, 46, 617-628 (1982).

9) S. S. Chiang, D. B. Marshall and A. G. Evans, J. Appl. Phys., 53, 298-311 (1982).

10) S. S. Chiang, D. B. Marshall and A. G. Evans, J. Appl. Phys., 53, 312-317 (1982).

11) R. F. Cook and G. M. Pharr, J. Am. Ceram. Soc., 73, 787-817 (1990).

12) M. Wada, H. Furukawa and K. Fujita, Proc. 10th Int. Congr. Glass, Jul. 8-13, Kyoto, Japan (1974) pp. 39-46.

13) W. B. Hillig, Proc. 6th Int. Congr. Glass, Jul. 8-14, Washington, U.S. (1962) p. 51.

14) J. E. Neely and J. D. Mackenzie, J. Mater. Sci., 3, 603609 (1968).
15) S. Yoshida, S. Isono, J. Matsuoka and N. Soga, J. Am Ceram. Soc., 84, 2141-2143 (2001).

16) J. D. Mackenzie, J. Am. Ceram. Soc., 46, 470-476 (1963).

17) S. Yoshida, J.-C. Sanglebœuf and T. Rouxel, J. Mater. Res., 20, 3404-3412 (2005).

18) S. Yoshida, H. Sawasato, T. Sugawara, Y. Miura and J. Matsuoka, J. Mater. Res., 25, 2203-2211 (2010).

19) S. Yoshida, M. Kato, A. Yokota, S. Sasaki, A. Yamada, J. Matsuoka, N. Soga and C. R. Kurkjian, J. Mater. Res., 25, 2291-2299 (2010).

20) P. Sellappan, T. Rouxel, F. Celarie, E. Becker, P. Houizot and R. Conradt, Acta. Mater., 61, 5949-5965 (2013).

21) C. Hermansen, J. Matsuoka, S. Yoshida, H. Yamazaki, Y. Kato and Y. Z. Yue, J. Non-Cryst. Solids, 364, 40-43 (2013).

22) S. Yoshida, J. Non-Cryst. Solids: X, 1, 100009 (2019).

23) S. Yoshida, Y. Hayashi, A. Konno, T. Sugawara, Y. Miura and J. Matsuoka, Phys. Chem. Glasses, 50, 6370 (2009).

24) R. Limbach, A. Winterstein-Beckmann, J. Dellith, D. Möncke and L. Wondraczek, J. Non-Cryst. Solids, 417418, 15-27 (2015).

25) M. Barlet, J. M. Delaye, T. Charpentier, M. Gennisson, D. Bonamy, T. Rouxel and C. L. Rountree, J. NonCryst. Solids, 417-418, 66-79 (2015).

26) G. Scannell, D. Laille, F. Célarié, L. Huang and T. Rouxel, Front. Mater., 4, 6 (2017).

27) Y. Kato, $\mathrm{Ph}$. D. thesis [in Japanese], The University of Shiga Prefecture, Japan (2011).

28) Y. Kato, H. Yamazaki, S. Yoshida and J. Matsuoka, J. Non-Cryst. Solids, 356, 1768-1773 (2010).

29) K. Januchta, R. E. Youngman, A. Goel, M. Bauchy, S. L. Logunov, S. J. Rzoska, M. Bockowski, L. R. Jensen and M. M. Smedskjaer, Chem. Mater., 29, 5865 5876 (2017).

30) C. Martinet, M. Heili, V. Martinez, G. Kermouche, G. Molnar, N. Shcheblanov, E. Barthel and A. Tanguy, J. Non-Cryst. Solids, 533, 119898 (2020).

31) C. L. Rountree, D. Vandembroucq, M. Talamali, E. Bouchaud and S. Roux, Phys. Rev. Lett., 102, 195501 (2009).

32) T. Sato, N. Funamori and T. Yagi, J. Appl. Phys., 114, 103509 (2013)

33) E. J. Frankberg, J. Kalikka, F. García Ferré, L. JolyPottuz, T. Salminen, J. Hintikka, M. Hokka, S. Koneti, T. Douillard, B. Le Saint, P. Kreiml, M. J. Cordill, T. Epicier, D. Stauffer, M. Vanazzi, L. Roiban, J. Akola, F. Di Fonzo, E. Levänen and K. Masenelli-Varlot, Science, 366, 864-869 (2019).

34) K. Zeng and D. J. Rowcliffe, Acta Metall. Mater., 43, 1935-1943 (1995).

35) T. Nagae, H. Kitahara, K. Ikeda, F. Yoshida, H. Nakashima, S. Ito and H. Abe, J. Ceram. Soc. Jpn., 110, 834-839 (2002).

36) F. C. Serbena and E. D. Zanotto, J. Non-Cryst. Solids, 358, 975-984 (2012).

37) G. Pezzotti and A. Leto, Phys. Rev. Lett., 103, 175501 (2009).

38) A. J. Dalladay and F. Twyman, Trans. Opt. Soc., 23, 165-169 (1922).

39) H. Aben and C. Guillemet, "Photoelasticity of Glass", 
Springer, Berlin Heidelberg (1993) pp. 86-101.

40) R. Oldenbourg and G. Mei, J. Microsc., 180, 140-147 (1995).

41) S. Yoshida, S. Iwata, T. Sugawara, Y. Miura, J. Matsuoka, A. Errapart and C. R. Kurkjian, J. NonCryst. Solids, 358, 3465-3472 (2012).

42) K. Asai, S. Yoshida, A. Yamada, J. Matsuoka, A. Errapart and C. R. Kurkjian, Mater. Trans., 60, 14231427 (2019).

43 ) D. B. Marshall and B. R. Lawn, J. Mater. Sci., 14, 2001-2012 (1979).

44) B. R. Lawn, T. P. Dabbs and C. J. Fairbanks, J. Mater. Sci., 18, 2785-2797 (1983).

45) M. Sakai, N. Hakiri and T. Miyajima, J. Mater. Res., 21, 2298-2303 (2006).

46) T. Miyajima and M. Sakai, Philos. Mag. A, 86, 57295737 (2006).

47) W. C. Oliver and G. M. Pharr, J. Mater. Res., 7, 1564 1583 (1992).

48) S. Yoshida, M. Kato, A. Yokota, S. Sasaki, A. Yamada, J. Matsuoka, N. Soga and C. R. Kurkjian, J. Mater. Res., 30, 2291-2299 (2015).

49) M. Sakai, J. Mater. Res., 14, 3630-3639 (1999).

50) Y. Y. Lim and M. M. Chaudhri, Mech. Mater., 38, 1213-
1224 (2006).

51) B. R. Lawn, J. Mater. Res., 19, 22-29 (2004).

52) S. Yoshida, K. Wada, T. Fujimura, A. Yamada, M. Kato, J. Matsuoka and N. Soga, Frontiers in Materials, 3, 54 (2016).

53) O. Benzine, S. Bruns, Z. Pan, K. Durst and L. Wondraczek, Adv. Sci., 5, 1800916 (2018).

54) H. Tran, S. Clément, R. Vialla, D. Vandembroucq and B. Rufflé, Appl. Phys. Lett., 100, 231901 (2012).

55) S. Fuhrmann, R. Limbach, C. Krywka and L. Wondraczek, Abs. SGT Cent. Conf., Sep. 4-8, Sheffield, U.K. (2016).

56) Y. Sung, S. Yoshida, Y. Kato and C. R. Kurkjian, J. Am. Ceram. Soc., 102, 5866-5872 (2019).

57) T. Lacondemine, J. Réthoré, É. Maire, F. Célarié, P. Houizot, C. Roux-Langlois, C. M. Schlepütz and T. Rouxel, Acta. Mater., 179, 424-433 (2019).

58) S. Yoshida, T. H. Nguyen, A. Yamada and J. Matsuoka, Mater. Trans., 60, 1428-1432 (2019).

59) Y. B. Gerbig and C. A. Michaels, J. Non-Cryst. Solids, 530, 119828 (2020).

$60)$ R. J. Hemley, H. K. Mao, P. M. Bell and B. O. Mysen, Phys. Rev. Lett., 57, 747-750 (1986).

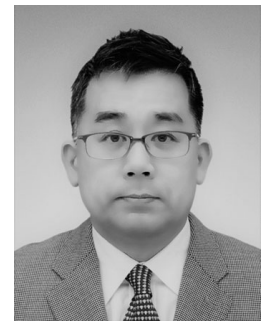

Satoshi Yoshida has just started his new career in April 2020 as a senior manager of Materials Integration Laboratories, AGC Inc., Japan. He received his B. E. (1993), M. E. (1995), and Doctor degree in Human and Environmental Studies (2003) from Kyoto University, Japan. In 1995, he started to work as an assistant professor of Department of Materials Science in the University of Shiga Prefecture (USP), Japan. From 2007 to 2020, he worked as an associate professor at USP. During the year 2004-2005, he also worked as a visiting professor of the University of Rennes 1, France. Dr. Satoshi Yoshida was awarded the 14th Otto Schott Research Award (2016) from Ernst Abbe Fund. His main research topic is deformation and fracture behavior of oxide glasses. 